Patricia Fernández Puente: None declared, Cristina Ruiz-Romero: None declared, Juan D. Cañete: None declared, Antonio Julià: None declared, Antonio Fernández Nebro: None declared, Jesús Tornero: None declared, Sara Marsal: None declared, Francisco J. Blanco Consultant for: AbbVie, Bioiberica, BMS, GSK, Grünenthal, Janssen, Lilly, Pfizer, Regeneron, Roche, Sanofi, TRB Chemedica, and UCB

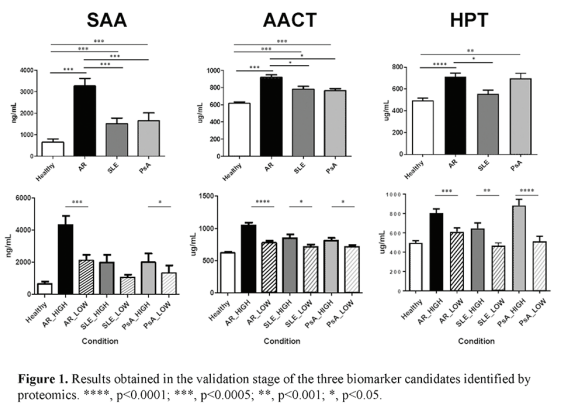

DOI: 10.1136/annrheumdis-2019-eular.2801

\section{THU0219 THE INVOLVEMENT OF MITOCHONDRIAL ACTIVATION VIA GLUTAMINOLYSIS IN HUMAN B CELL DIFFERENTIATION AND ITS RELEVANCE TO THE PATHOGENESIS OF SLE}

Maiko Hajime ${ }^{1}$, Shigeru Iwata ${ }^{1}$, Mingzeng Zhang ${ }^{1}$, Hiroko Miyata ${ }^{1}$

Seunghyun Lee ${ }^{1}$, Shingo Nakayamada ${ }^{1}$, Kazuo Yamamoto $^{2}$, Yosuke Okada ${ }^{1}$, Yoshiya Tanaka'. 'University of Occupational and Environmental Health, Kitakyushu, Japan; ${ }^{2}$ Nagasaki University School of Medicine, Biomedical Research Support Center, Nagasaki, Japan

Background: B cells play a crucial role in Systemic Lupus Erythematosus (SLE). Recently, "Immunometabolism" attract much attention. Glucose and glutamine are important nutrition for energy production such as ATP in various cells. It has been reported that aerobic glycolysis, glutaminolysis and mitchondrial functions enhanced in cancer cells. However, the involvement of metabolic reprograming in plasmablast differentiation and its relevance to the pathogenesis of SLE remained elusive.

Objectives: We first investigated the abnormality of mitochondria in B cells from patients with SLE by flow cytometry. Next, B cells were isolated from healthy donors (HDs) and metabolic reprograming were assessed in vitro.

Methods: First, peripheral blood mononuclear cells (PBMCs) were obtained from age-matched $31 \mathrm{HDs}$ and 29 patients with SLE. The mitochondrial membrane potential was measured with DiOc6 by flow cytometry. In addition, $\mathrm{CD}_{19}{ }^{+}$cells were isolated from $\mathrm{HDs}$ and stimulated with CpG (TLR9 ligand) and IFN- $\alpha$. Change of aerobic glycolysis, glutaminolysis and mitochondrial functions were assessed in the absence of glucose or glutamine and in the addition of metformine, which is known as AMPK activator, in vitro.

Results: We first examined the abnormality of mitochondria in B cells from patients with SLE using DiOc6 as a marker of depolarization-activated mitochondrial membrane. Baseline characteristics of SLE were males: females=1:28, age 40.2 years, disease duration 132.2 months, SLEDAI 6.4 and BILAG 6.2 at the timing of admission due to exacerbation of SLE. The percentage of $\operatorname{lgD}{ }^{-} \mathrm{CD}^{-} 7^{-}$memory $\mathrm{B}$ cells were higher than those of HDs, while the percentage of $\operatorname{lgM}$ memory B cells were lower than that of $\mathrm{HDs}$. The percentage of $\mathrm{CD}^{-} \mathrm{DiOc6}^{+}$cells in $\lg D^{-} C D 27^{-}$memory $B$ cells from patients with SLE were higher than that of HDs. These results indicate that B cells from patients with SLE have the abnormality of differentiation and mitochondrial functions. Next, we assessed the change of aerobic glycolysis, mitochondrial functions and glutaminolysis in the process of plasmablast differentiation in vitro. Stimulation with $\mathrm{CpG}$ and IFN- $\alpha, 1$ ) enhanced aerobic glycolysis, which was assessed by lactate production. 2) increased the area of cytoplasm including many expanded mitochondrial cristae with slightly wider and loosely organized intermembrane space in electric microscopy, accompanied with ROS production and DiOc6 up-regulation, 3) induced $\mathrm{CD} 27^{\mathrm{hi}} \mathrm{CD} 38^{\mathrm{hi}}$ plasmablasts differentiation and immunoglobulin production. Lactate production was decreased in the absence of both glucose or glutamine. ROS production and DiOc6 expression were decreased in the absence of glutamine, leading to inhibition of plasmablasts differentiation and immunoglobulin production. On the other hand, this tendency was not shown in the absence of glucose. Next, we evaluated oxygen consumption rate (OCR). OCR was also suppressed in the absence of glutamine. Metformin, abrogated glutamine uptake, resulting in suppression of ROS production, DiOc6 expression, plasmablast differentiation and immunoglobulin production.

Conclusion: These results suggest that mitochondrial activation via glutaminolysis may play an important role in the differentiation from IgD CD27 double negative $\mathrm{B}$ cells to plasmablasts and production of immunoglobulins in patients with SLE.

Disclosure of Interests: Maiko Hajime: None declared, Shigeru Iwata: None declared, Mingzeng Zhang: None declared, Hiroko Miyata: None declared, SEUNGHYUN LEE: None declared, Shingo Nakayamada Grant research support from: Mitsubishi-Tanabe, Takeda, Novartis and MSD, Speakers bureau: Bristol-Myers, Sanofi, Abbvie, Eisai, Eli Lilly, Chugai, Asahi-kasei and Pfizer, Kazuo Yamamoto: None declared, Yosuke Okada: None declared, Yoshiya Tanaka Grant/research support from: Abbvie, Astellas, Bristol-Myers Squibb, Chugai, Daiichi-Sankyo, Eisai, MitsubishiTanabe, MSD, Ono, Taisho-Toyama, Takeda, Speakers bureau: Abbvie, Asahi-kasei, Astellas, Bristol-Myers Squibb, Chugai, Daiichi-Sankyo, Eli Lilly, Eisai, Glaxo-Smithkline, Janssen, Mitsubishi-Tanabe, Novartis, Pfizer Japan Inc, Sanofi, Takeda, UCB, YL Biologics DOI: 10.1136/annrheumdis-2019-eular.3677

\section{THU0220 PEPTIDYLARGININE DEIMINASE 4 DEFICIENCY AMELIORATED A MURINE MODEL OF LUPUS BY REDUCING NEUTROPHIL MIGRATION TO THE KIDNEY}

Norio Hanata ${ }^{1}$, Hirofumi Shoda ${ }^{1}$, Hiroaki Hatano ${ }^{1}$, Yasuo Nagafuchi ${ }^{1}$,

Toshihiko Komai ${ }^{1}$, Tomohisa Okamura ${ }^{1,2}$, Akari Suzuki $^{3}$, Kazuhiko Yamamoto $^{3}$, Keishi Fujio ${ }^{1}{ }^{1}$ Department of Allergy and Rheumatology, Graduate School of Medicine, The University of Tokyo, Tokyo, Japan; ${ }^{2}$ Department of Functional Genomics and Immunological Diseases, Graduate School of Medicine, The University of Tokyo, Tokyo, Japan; ${ }^{3}$ Laboratory for Autoimmune Diseases, RIKEN Center for Integrative Medical Sciences, Yokohama, Japan

Background: Peptidylarginine deiminase 4 (PADI4) is reported to play several biological roles in neutrophils, including neutrophil extracellular trap formation, apoptosis, and epigenetic regulation. Neutrophils could play pivotal roles in the pathogenesis of SLE.

Objectives: We set to determine the pathological role of neutrophils and PADI4 in SLE using Padi4 knock out (KO) mice.

Methods: A murine model of imiquimod (IMQ)-induced lupus was analyzed. A TLR7 agonist, IMQ, was administered topically to the ear skin of B6 wild-type (WT) and Padi4 KO mice. Proteinuria, spleen weight, serum anti-dsDNA level, frequencies of spleen and renal immune cells, and the histopathological findings of ear skin and kidney, were assessed. Neutrophil migration and adhesion were evaluated by adoptive transfer experiments in vivo, and adherence assays in vitro

Results: Compared with IMQ-treated WT (WT-IMQ) mice, Padi4 KO-IMQ mice showed decreased spleen weight. Moreover, proteinuria, and dermatitis were not exacerbated in the Padi4 KO-IMQ mice. There was a positive correlation between the frequency of kidney neutrophils and the degree of proteinuria, while significant decreases in kidney neutrophils were noted in the Padi4 KO-IMQ mice. Notably, the elevation of serum anti-dsDNA levels, or the degree of immune complex deposition in the kidney, showed no significant difference between the WT-IMQ and Padi4 KO-IMQ mice. In the adoptive transfer experiment, there was a significant decrease in the transferred Padi4 KO neutrophil infiltrations in the kidney compared with those of WT neutrophils. The frequency of adhesive neu trophils toward ICAM-1 was significantly decreased in the Padi4 KO neutrophils primed by the TLR7 agonist.

Conclusion: Nephritis was ameliorated in Padi4 KO-IMQ mice. Our study shed light on the importance of neutrophils in the pathogenesis of SLE, and the suppression of neutrophil function by inhibition of PADI4 will be a unique therapeutic strategy for SLE.

Disclosure of Interests: Norio Hanata: None declared, Hirofumi Shoda Speakers bureau: Astellas, Abbie, BMS, GSK, Daiichi-Sankyo, Pfizer Chugai, UCB, Eli Lilly., Hiroaki Hatano: None declared, Yasuo Nagafuchi Grant/research support from: BMS, Chugai., Speakers bureau: BMS, Pfizer, Kissei., Toshihiko Komai Grant/research support from: GlaxoSmithKline., Tomohisa Okamura Grant/research support from: Chugai, Novartis., Akari Suzuki Grant/research support from: Takeda, Chugai, Pfizer., Kazuhiko Yamamoto Grant/research support from: Astellas, BMS Daiichi-Sankyo, Mitsubishi Tanabe, Pfizer, Ayumi, Takeda, Chugai, Eisai, Taisho Toyama, UCB, Janssen, Eli Lilly, and NIPPON KAYAKU., Speak ers bureau: Astellas, BMS, Daiichi-Sankyo, Mitsubishi Tanabe, Pfizer, Ayumi, Takeda, Chugai, Eisai, Taisho Toyama, UCB, Janssen, Eli Lilly, and NIPPON KAYAKU., Keishi Fujio Grant/research support from: Astellas, BMS, Daiichi-Sankyo, Mitsubishi Tanabe, Pfizer, Ayumi, Takeda, 
Chugai, Eisai, Taisho Toyama, UCB, Janssen, Eli Lilly, and NIPPON KAYAKU., Speakers bureau: Astellas, BMS, Daiichi-Sankyo, Mitsubishi Tanabe, Pfizer, Ayumi, Takeda, Chugai, Eisai, Taisho Toyama, UCB, Janssen, Eli Lilly, and NIPPON KAYAKU. DOI: 10.1136/annrheumdis-2019-eular.4714

\section{THU0221 DRY EYE IN SJÖGREN'S SYNDROME: CHEMOKINE AND CYTOKINE TEAR SPECTRUM}

Gabriela Hernandez-Molina, Narlly Ruiz-Quintero, Guadalupe Lima, Diego Hernández-Ramírez, Amaya Llorente-Chavez, Vanessa SaavedraGonzález, Rodolfo Jimenez-Soto, Luis Llorente. Instituto Nacional de Ciencias Médicas y Nutrición Salvador Zubirán, Mexico City, Mexico

Background: Previous studies have linked the participation of multiple chemokines and cytokines in the physiopathology of primary Sjögren's syndrome (PSS), however data regarding their presence in tears is scarce

Objectives: To evaluate a panel of chemokines/cytokines in the tears of patients with PSS and correlate them with ocular symptoms as well as objective ocular tests.

Methods: We included 21 patients with PSS (EULAR/ACR criteria). A single expert ophthalmologist in dry eye evaluated the patients and assessed the tear film break-up time, Schirmer-I test, tear meniscus height, the Van Bijsterveld staining score and the SICCA Ocular Staining Score (OSS). We classified lacrimal dysfunction severity in two categories ( $1=$ mild, mild $/$ moderate or moderate, and $2=$ moderate/severe and severe). We scored the ESSPRI, and ocular dryness VAS as well as the Ocular Surface Disease Index (OSDI), a 12-item scale for the assessment of symptoms related to dry eye disease and their effect on vision. Tear samples were collected using sterile tear flow strips, that were immediately frozen at $-86^{\circ} \mathrm{C}$ until assayed. Once defrosted, the tears were extracted from the strips using a buffer containing $0.5 \mathrm{M} \mathrm{NaCl}$ and $0.5 \%$ Tween-20. We tested IFN- $\gamma$, IL-10, IL-12, IL-17A, IL-1 $\beta$, IL-2, IL-21, IL-23, IL-5, IL-6, IL-8, TNF- $\alpha$, BAFF, CXCL10 and CCL2 by Luminometry. We also included 21 healthy controls without dry eye, to test chemokines/ cytokines that after our initial screening were meaningful.

Results: Most patients were females (90.4\%), mean age $59.3 \pm 13$ years and median disease duration 7.9 years $(0.5-27)$. All of them had ocular and oral symptoms. The median tear film break-up time was 6 seconds (2-9), median Schirmer-I test $6 \mathrm{~mm}$ (1-25), median lacrimal meniscus height $1.5 \mathrm{~mm}(0.5-2)$, median Van Bijsterveld staining score 10 points (2-18), median OSS 7 points (2-11), median ESPPRI score 6.7 points (29.2) and median ocular dryness EVA score 9 points (1-10).

We did not detect most of the evaluated chemokines/cytokines with the exception of IL-8, CXCL10, and CCL2. The former was similar in both, patients and controls. PSS patients had lower levels of CXCL10 (472.8 $\mathrm{pg} / \mu \mathrm{L}$ vs $1652 \mathrm{pg} / \mu \mathrm{L}, \mathrm{p}=0.01)$ and $\mathrm{CCL} 2$ (1.08 $\mathrm{pg} / \mu \mathrm{L}$ vs $9 \mathrm{pg} / \mu \mathrm{L}$ ) than controls. Indeed, patients with worst lacrimal dysfunction severity had the lowest levels of CXCL10 (239.3 pg/ $\mu \mathrm{L}$ vs. $646.2 \mathrm{pg} / \mu \mathrm{L}, \mathrm{p}=0.02)$. We found correlations among $\mathrm{CXCL} 10$ and $\mathrm{CCL} 2(\mathrm{~T}=0.30, \mathrm{p}=0.02)$ and lacrimal meniscus height $(T=0.55, p=0.005)$, as well as with CCL2 and lacrimal meniscus height $(T=0-57, p=0.01)$. None of the other variables were correlated.

Conclusion: We identified CXCL10 and CCL2 as the main chemokines in tears of patients with PSS. CXCL10 seems to participate in the normal eye homeostasis.

Disclosure of Interests: None declared

DOI: 10.1136/annrheumdis-2019-eular.5226

\section{THU0222 PLASMACYTOID DCS FROM PATIENTS WITH SJÖGREN'S SYNDROME ARE TRANSCRIPTIONALLY PRIMED FOR ENHANCED PRO-INFLAMMATORY CYTOKINE PRODUCTION}

Maarten Hillen ${ }^{1}$, Aridaman Pandit ${ }^{1}$, Sofie Blokland ${ }^{1}$, Sarita Ay Hartgring ${ }^{1}$, Cornelis Bekker ${ }^{1}$, Eefje van der Heijden ${ }^{1}$, Nila Servaas ${ }^{1}$, Marzia Rossato ${ }^{1}$, Aike A. Kruize ${ }^{2}$, Joel van Roon ${ }^{1}$, Timothy R. Radstake ${ }^{2} .{ }^{1}$ University Medical Centre Utrecht, Laboratory of Translational Immunology/Rheumatology and Clinical Immunology, Utrecht, Netherlands; ${ }^{2}$ University Medical Centre Utrecht, Rheumatology and Clinical Immunology, Utrecht, Netherlands

Background: Type-I IFN activity is associated with pathogenesis and increased disease activity in primary Sjögren's syndrome (pSS). In addition, deficiency for the type-I IFN receptor in mice prevents experimentalSjögren's syndrome. Plasmacytoid dendritic cells (pDC) are the premier type-I IFN producing immune cells and aberrances in their functional properties may underlie pSS immunopathology. Assessing the molecular basis of this may provide a better understanding of pSS pathogenesis and new opportunities for therapeutic intervention.

Objectives: To delineate the dysregulation of pSS pDCs using RNA sequencing and compare their transcriptional profile to pDCs obtained from patients with non-Sjögren's sicca (nSS) and healthy controls (HC).

Methods: All pSS patients met the classification criteria. nSS patients presented with dryness complaints without a known cause, did not have any generalized autoimmune disease including pSS as evaluated by an experienced rheumatologist, and did not fulfil the classification criteria. pSS $(n=25)$, nSS $(n=20)$, and HC $(n=17)$ donors were included in two independent cohorts $(n=31$ each). Circulating BDCA-4 expressing pDCs were isolated and RNA-sequencing was performed, after which data-driven networks and modular analysis were used to identify signatures of consistently differentially-expressed genes. pSS and $\mathrm{HC}$ pDCs were cultured in the presence of endosomal TLR ligands, after which gene expression and secreted cytokine levels were measured.

Results: We identified signatures of consistently co-expressed and differentially expressed genes that indicated transcriptional activation in patien pDCs, which was remarkably reproducible in two independent cohorts These included a type-I IFN-associated signature, a ribosomal protein sig nature, and a transcriptional machinery signature. Corroborating the transcriptomic profile, stimulated pSS pDCs produced higher levels of typeinterferon upon in vitro stimulation. nSS patients formed an intermediate group in which some patients were molecularly similar to pSS patients. Finally, we developed a discriminative classifier on the basis of the identified transcriptional profiles that discriminated pSS patients from $\mathrm{HC}$ with $100 \%$ sensitivity and $80 \%$ specificity, and identified a group of pSS-like patients within the nSS group.

Conclusion: Circulating pSS pDCs exhibit a transcriptional signature similar to activated pDCs and are primed for enhanced production of proinflammatory cytokines, including type-I IFN. Our data provide in-depth characterization of the aberrant regulation of pSS pDCs and substantiate their perceived role in the immunopathology of pSS and other type-I interferon-associated autoimmune diseases.

Disclosure of Interests: None declared

DOI: 10.1136/annrheumdis-2019-eular.7019

\section{THU0223 CHRONIC ADRENERGIC STIMULATION OF MINOR SALIVARY GLANDS OF PATIENTSWITH PRIMARY SJÖGREN'S DRIVES ER STRESS AND ACTIVATION OF THE UNFOLDED PROTEIN RESPONSE}

Kalliopi Moustaka ${ }^{1}$, Stergios Katsiougiannis ${ }^{1}$, Roxane Tenta ${ }^{1}$, Sofia Havaki ${ }^{2}$ Pari Koutsoudaki ${ }^{2}$, Haralampos M. Moutsopoulos ${ }^{3}$, Fotini Skopouli ${ }^{1}$. Harokopio University, Nutrition and Clinical Dietetics, Ko $\alpha \lambda \lambda_{1} \theta \dot{\varepsilon} \alpha$, Greece; ${ }^{2}$ National and Kapodistrian University of Athens, Histology and Embryology, Athens, Greece; ${ }^{3}$ The Academy of Athens, Athens, Greece

Background: Sjögren's syndrome (SS) is a common autoimmune disease in which the main targets of immune injury are specific secretory epithelia, such as salivary and lacrimal glands. Stress appears to play a significant role in the initiation of this entity.

Objectives: The aim of the present study was to investigate whether chronic stress plays a role in triggering endoplasmic reticulum (ER) stress in salivary gland epithelial cells from SS patients.

Methods: Minor salivary gland biopsy specimens were obtained from six SS patients and six control patients with sicca symptoms not fulfilling AECG criteria [1]. The expression and cellular localization of $\beta 1-, \quad \beta 2$ and $\alpha 1$-adrenoceptors and the levels of cAMP were measured by immunofluorescence. The morphology of the ER was evaluated in situ by Transmission Electron Microscopy (TEM). Primary salivary gland epithelial cell lines (SGEC) derived from minor salivary gland biopsies, were established by the explant out-growth technique [2] and were treated with epinephrine and norepinephrine. The protein levels of the ER stress markers GRP78/Bip and C/EBP homologous protein (CHOP) were determined by immunoblot analysis.

Results: In situ immunofluorescence staining revealed increased expression of $\beta 1-, \beta 2$ - and $\alpha 1$ adrenoceptors as well as cAMP levels in tissues derived from SS patients compared to controls. TEM evaluation of salivary tissues from SS subjects revealed extensive dilation of the ER lumen compared to controls. Treatment of SGEC with epinephrine and norepinephrine did not influence cell survival (cell viability assay). To mimic chronic stress in vitro, epinephrine was applied for 10 days on SGEC. It was found that $20 \mu \mathrm{M}$ Epinephrine induced severe ER stress on SGEC, as attested by increased expression of GRP78/Bip and CHOP, after 3 and 6 days of treatment. The expression of ER stress markers returned to basal levels after 10 days of treatment. 